\title{
Pop, Songs und Philologie
}

\section{Ein Editorial}

\author{
Gerhard Kaiser · Kai Sina
}

Online publiziert: 6. Juli 2016

(C) J.B. Metzler Verlag GmbH, Stuttgart 2016

»We learned more from a three minute record, baby, than we ever learned in school«, heißt es im Song No Surrender von Bruce Springsteen. Die Jugendlichen, von denen der Song handelt, seien, so das erzählende Ich im ersten Vers, aus ihrer Schulklasse ausgebrochen, um von all den uneingeweihten Idioten wegzukommen: »to get away from these fools«.

Soweit eine der philisterkritischen Basiserzählungen aus dem reichhaltigen Archiv der Popmythologie; einer Popmythologie, die von Beginn an immer auch schon eine anti-bildungsbürgerliche war, man denke nur an Chuck Berrys Roll Over Beethoven. Aber wie es sich mit den Bildungsinstitutionen - Schulen, Hochschulen nun einmal verhält: Sie sind meist zählebiger und anpassungsfähiger, als selbst die Agilsten unter ihren Verächtern. Wenn die Bildungsflüchtigen mit Pop im Tornister nur lange genug ihre Lieder vom Leben im Hier und Jetzt gesungen haben, dann werden diese Lieder selbst Geschichte, und die Akademien holen in ihre Seminare, Vorlesungen und Veröffentlichungen eben jene Lieder zurück, die einst von ihrer Überlebtheit sangen.

Seit Carl Perkins 1956 und ein Jahr später Elvis Presley die blauen Velourslederschuhe zur unbetretbaren Zone erklärten, sind fast sechzig Jahre vergangen, und die Wissenschaften sind dem Pop seitdem immer wieder auf die Füße getreten - und dies, obwohl das Verhältnis der Verachtung zwischen Pop und Wissenschaft durchaus ein wechselseitiges war. Aber mehr als siebzig Jahre sind auch vergangen, seit die Kritische Theorie sämtliche Erscheinungen des Populären als kulturindustrielle Massenbespaßungsprodukte verdammte; als Produkte, die ihre Konsumenten bei

G. Kaiser $(\bowtie) \cdot$ K. Sina

Universität Göttingen, 37073 Göttingen, Deutschland

E-Mail: Gerhard.Kaiser@phil.uni-goettingen.de

K. Sina

E-Mail: kai.sina@phil.uni-goettingen.de 
Laune und im Takt (der Selbstausbeutungsbereitschaft) halten, indem sie über die wahren Entfremdungsverhältnisse in kapitalistischen Gesellschaften den Zuckerguss des temporären Vergnügens streuen. Die einst wie in Stein gemeißelten Sentenzen der Kritischen Theorie - >Fun ist ein Stahlbad<, >Vergnügtsein heißt Einverstandensein< - eigneten sich mittlerweile fast zum T-Shirt-Aufdruck und wären somit selbst zu jenem Pop geworden, vor dem die amtlich zornigen Frankfurter immer gewarnt haben.

Im Gefolge von Leslie Fiedlers sprichwörtlich gewordener Aufforderung, den Graben zwischen vermeintlich hoher, ernster und vermeintlich niederer Unterhaltungskultur zu schließen, im Gefolge auch der popsoziologischen Arbeiten im Umfeld der Cultural Studies (John Fiske, Simon Frith) bis hin schließlich zu den poptheoretischen Überlegungen eines Peter Wicke oder Diedrich Diederichsen, der mit seinem Buch Über Pop-Musik (2014) die gewichtige Summe seiner bisherigen poptheoretischen Arbeiten vorgelegt hat, konnte sich Pop als fester und expandierender Gegenstandsbereich im akademischen Feld etablieren.

Dies scheint jedoch ein Prozess zu sein, auf den die Künstler und die Popindustrie ihrerseits reagieren: Der Pop tritt in sein historisch-kritisches Stadium ein. Bob Dylans 1991 unter dem Titel Bootleg Series begonnene Veröffentlichung von bisher unveröffentlichten Liedern und/oder alternativen Varianten von bereits veröffentlichten Songs hat es mittlerweile auf zwölf Folgen gebracht. Und sie markiert lediglich den Anfang. So präsentiert sich etwa - um nur zwei weitere Beispiele zu nennen das Booklet zu Leonard Cohens 2012 erschienenem Spätwerk Old Ideas wie eine Art stilisierter Dichternachlass, mit handschriftlichen Vorstufen zu Songtexten, Notizen und Zeichnungen, gerade so, als wolle der alte Sänger nunmehr die Phase der philologischen Erschließung seines Gesamtwerks einleiten. Vergleichbares lässt sich bei Neil Young beobachten, der vor einigen Jahren sein gesammeltes Songmaterial der Jahre 1963 bis 1972 veröffentlicht hat, verpackt in Archivkisten und beschriftet mit dem entsprechenden Titel: Archives. Ein dergestalt klares Nachlassbewusstsein war bisher eigentlich nur aus dem Bereich der Literatur bekannt.

Von dieser neuen, gegenseitigen Nähe zwischen Pop und Akademie zeugt auch die vorliegende Ausgabe der Zeitschrift für Literaturwissenschaft und Linguistik, die sich ganz dem Song und damit - um eine Definition Diederichsens aufzugreifen - der »kleinsten signifikanten oder relevanten Einheit der Pop-Musik « widmet. Songs sind deshalb »signifikant« oder »relevant«, weil sie uns von der Wiege bis zur Bahre, vom Schlaflied und nursery song über das Liebes- und Kriegslied bis zur Totenklage begleiten. Sie bilden als Gebrauchskunstwerke im wahrsten Sinne des Wortes jene Schnittstellen, an denen die Kunst ins Leben umschlägt - ein alter Intellektuellentraum. Songs sind gesungene, bisweilen tanzbare Literatur, das heißt, sie affizieren in ihrer Mischung aus Musik, Stimme, Text und Performanz den ganzen Menschen. In dieser Hinsicht bieten sie mitunter vielleicht sogar mehr und anderes als reine Schrifttexte. Die wichtigsten, gesellschaftlichen Funktionen der geschriebenen Literatur teilen sie jedenfalls allemal: Auch Songs fungieren als Speicher- und Affekterregungsmedien, als Seismografen, als Kompensations- und Distinktionsvehikel und als Medien zum Aufbau von Gegenwelten.

Gleichwohl mag eine solche Sammlung literaturwissenschaftlicher Annäherungen, wie sie hier vorliegt, mit einigen Widerständen und Einwänden rechnen. Nur 
einige der offensichtlichsten sollen hier aufgegriffen werden, und wir wollen zumindest kurz umreißen, warum wir uns - wenngleich wir solche Einwände vielleicht nicht völlig entkräften können - dazu entschieden haben, dieses Projekt dennoch auf den Weg zu bringen.

Erstens. Der sicherlich allgemeinste Einwand betrifft zwar den analytischen Umgang mit künstlerischen Artefakten überhaupt (und eben nicht nur den wissenschaftlichen Umgang mit Songs), wird aber in diesem Kontext besonders gerne angeführt: Songs mag man oder man mag sie nicht, sie ergreifen oder sie lassen einen kalt. Muss man darüber überhaupt noch reden? Zerstört nicht der analytische Zugriff gerade das Lebendige und Wirkungsvolle jenes Phänomens, das zu ergründen er vorgibt? Freilich, man muss nicht. Aber, so zumindest unser Dafürhalten, man kann. Gute Songs halten das aus, sie werden nicht schlechter dadurch, dass man über sie spricht, im Gegenteil, vielleicht stimmt die alte Formel in diesem Fall ja doch: Dass man >begreifen< will, >was einen ergreift<, und dass die Anstrengungen des Begriffs das Erleben noch steigern können.

Zweitens. Darf man einen Zeitschriftenband über populäre Songs ohne Berücksichtigung der Beatles, der Rolling Stones, Joni Mitchells, Neil Youngs oder Janis Joplins überhaupt veranstalten? Schon der bewusst offene Titel des vorliegenden Heftes antwortet auf diesen naheliegenden Vorwurf mit einem entschiedenen Bekenntnis: Die Auswahl der Stücke, die hier analysiert werden, folgt nicht oder zumindest nicht in Gänze dem popkulturellen Kanon, oder besser: einem von vielen Kanones. Denn was verbindet schon die popmusikalische Universalbibliothek des Reclam-Verlags mit den grellen Shows der ultimativen Hits, wie sie allabendlich im Privatfernsehen zu sehen sind? Und welcher Weg führt von hier aus wiederum zu den zahllosen Best-of-Listen der Musikzeitschriften, die ihrerseits unterschiedlichste soziale Gruppen mit ihren je eigenen Neigungen ansprechen, vom Liebhaber des abgehangenen Rock- und Pop-Klassikers, der vermutlich zum Rolling Stone greifen wird, bis zum distinktionsverliebten Akademiker mit seiner poptheoretisch geschulten Spex? Bereits in diesen wenigen Befunden zeigt sich, dass die Kanones im Bereich der Popmusik, noch sehr viel stärker als im Bereich der Literatur, einer milieuspezifischen Ausdifferenzierung unterliegen, die sich in einer Aufsatzsammlung wie dieser niemals angemessen abbilden ließe. Mit der Entscheidung, den Beiträgern und Beiträgerinnen dieses Heftes selbst die Auswahl ihrer Gegenstände zu überlassen, haben wir versucht, diesem Sachverhalt Rechnung zu tragen. Im Ergebnis hat dies zu einer erfreulich vielstimmigen Zusammenstellung jenseits des allzu Erwartbaren geführt - eine Auswahl, die von Édith Piaf und Velvet Underground über Víctor Jara und Queen bis Wir sind Helden mehr als fünfzig Jahre Popgeschichte umfasst, die den Begriff des Populären ausdehnt von der Jugendkultur bis hinein ins juste milieu und dabei zugleich deutlich internationale Züge trägt, was dem Gegenstand freilich nur angemessen ist.

Drittens und zuletzt stellt sich die Frage, warum das Medium des Songs, zumal des aufgeführten Songs in seinem plurimedialen Zusammenspiel aus Musik, Text, Stimme und so fort, ausgerechnet aus literaturwissenschaftlicher Perspektive angemessen zu entschlüsseln sein sollte. Ohne uns die je eigenen Kompetenzen einer popkulturell interessierten Geschichtswissenschaft, Kulturanthropologie, Kunstsoziologie oder gar Musikwissenschaft anmaßen zu wollen: Songs lassen sich doch, 
wie andere audiovisuelle Medienprodukte, auch als Zeichensysteme verstehen, die sich bis zu einem gewissen Grad (und dieser Grad muss jeweils mitbedacht werden) als Texte begreifen lassen; Texte hier nun allerdings nicht im engeren Sinne verstanden bloß als Wort und Schrift, sondern im weiteren Sinne als zeichenhafte Konstellationen, die über das geschriebene oder gesprochene Wort hinaus in Ritualen, Theater, Gebärden, Festen oder eben in Gesängen, Konzerten, Musikvideos usw. verkörpert sind.

Wer nun befürchtet, der Literaturwissenschaft komme im Zuge einer dergestalt kulturwissenschaftlichen Erweiterung des Blickfelds ihr eigentlicher Gegenstand (vollends) abhanden, dem lässt sich aus fachgeschichtlicher Sicht entgegnen: Das Lied, verstanden als deutschsprachige Variante des Songs und des französischen Chansons, bildete lange Zeit geradezu den Kern einer der drei Hauptgattungen der Literaturwissenschaft, ja die Abtrennung der hochkulturellen Lyrik von der (vermeintlich) gemeinen Liedform ist eine Entwicklung, die wirklich nachhaltig erst in der zweiten Hälfte des 20. Jahrhunderts vollzogen worden ist. Eine gattungsgeschichtlich informierte Literaturwissenschaft muss diese Differenzierung zwar als ein historisches Faktum zur Kenntnis nehmen; ihr durch einen verengenden Zuschnitt ihres Gegenstandsbereiches hinterherzulaufen - das braucht sie aber nicht. Ermutigung hierfür gibt es an unterschiedlichen Stellen. Reizvoll erscheint etwa die von Greil Marcus und Werner Sollors herausgegebene New Literary History of America (2009), die Songs und Poems, Novels und Lyrics in vielstimmiger Weise (wieder) zusammenbringt.

Eine wichtige Mitanregung für dieses Projekt kommt vonseiten unserer Studierenden, die uns, im Rahmen von Seminaren sowie Bachelor- und Masterarbeiten, immer wieder Songs und besonders Songtexte als Untersuchungs- und Prüfungsgegenstände vorgeschlagen haben. Hieraus ging die Göttinger Ringvorlesung »13 Lieder. Lektüren und Analysen populärer Songs« (Wintersemester 2014/15) hervor - und aus ihr die Essays und Aufsätze des vorliegenden Heftes. 